TSUKUBA J. MATH.

Vol 6 No 2 (1982). 305-318

\title{
ON M-RECURSIVELY SATURATED MODELS OF ARITHMETIC
}

\author{
By
}

\author{
Akito TsuBoI
}

\section{Introducaion.}

In [6], C. Smorynski investigated the properties of models of arithmetic using the notions of recursive saturation and short recursive saturation. In this paper, we shall generalize these notions and obtain new isomorphism criteria (Theorems A and B) and embeddability criteria (Theorems D and E) for countable models of arithmetic.

Throughout, $\mathscr{P} \mathcal{A}$ denotes Peano arithmetic with the induction schema for all formulas in some finite language $L \supseteqq\left\{0,{ }^{\prime},+, \cdot\right\} . \Delta_{0}$ denotes the set of all quantifier bounded formulas in $L$. Let $M$ and $N$ be countable models of $\mathscr{P} \mathcal{A}$ with $M \subseteq N$. We say $N$ is $M$-recursively saturated ( $M^{s}$-recursively saturated) if $N$ realizes every (short) type $\tau$ which is $\Delta_{1}$ on $\mathbf{H F}_{M}$, where $\tau$ may contain countably many parameters from $M$. It can be easily shown that $M$-recursive saturation $\left(M^{s}\right.$-recursive saturation) corresponds with (short) recursive saturation, if $M=\left\langle\omega ; 0,{ }^{\prime},+, \cdot\right\rangle$. For $A \subseteq|N|, D f(N, A)$ denotes the set of all elements in $N$ which are definable in $N$ using parameters from $A$. We put:

$$
\begin{aligned}
& T h_{M}(N)=\left\{\phi\left(c_{a_{1}}, \cdots, c_{a_{n}}\right): a_{1}, \cdots, a_{n} \in|M| \text { and } N \vDash \phi\left(c_{a_{1}}, \cdots, c_{a_{n}}\right)\right\}, \\
& T h_{M}^{\Delta} \mathrm{o}(N)=\left\{\phi\left(c_{a_{1}}, \cdots, c_{a_{n}}\right): \phi \in \Delta_{0}, a_{1}, \cdots, a_{n} \in|M| \text { and } N \vDash \psi\left(c_{a_{1}}, \cdots, c_{a_{n}}\right)\right\},
\end{aligned}
$$

$S S_{M}^{\Delta}(N)=\{X \cap|M|: X$ is a subset of $|N|$ which is definable in $N$ using a $\Delta_{0}$-formula with parameters from $\left.|N|\right\}$.

Our main results of this paper are as follows:

THEOREM A. Suppose that $N_{1}$ and $N_{2}$ are $M$-recursively saturated countable models of $\mathscr{P} \mathcal{A}$ such that $T h_{M}\left(N_{1}\right)=T h_{M}\left(N_{2}\right)$ and $S S_{M^{0}}^{\Delta_{0}}\left(N_{1}\right)=S S_{M}^{\Delta}{ }^{0}\left(N_{2}\right)$. Then there is an isomorphism $f: N_{1} \rightarrow N_{2}$ which is identical on $M$.

THEOREM B. Suppose that $N_{1}$ and $N_{2}$ are $M^{s}$-recursively saturated models of $\mathscr{P} A$ such that $T h_{M}^{\Delta} 0\left(N_{1}\right)=T h_{M}^{\Delta} 0\left(N_{2}\right)$ and $S S_{M}^{\Delta} 0\left(N_{1}\right)=S S_{M}^{\Delta} 0\left(N_{2}\right)$. Suppose that both $N_{1}$ and $N_{2}$ are cofinal extensions of $M$. Then there is an isomorphism $f: N_{1} \rightarrow N_{2}$ 
which is identical on $M$.

THEOREM C. Suppose that $N_{1}$ and $N_{2}$ are countable cofinal extensions of $M$ with $T h_{M}^{\Delta 0}\left(N_{1}\right)=T h_{M}^{\Delta 0}\left(N_{2}\right)$. Then there are $M^{s}$-recursively saturated elementary cofinal extensions $N_{1}^{*}$ of $N_{1}$ and $N_{2}^{*}$ of $N_{2}$ such that $S_{M^{0}}^{\Delta_{0}}\left(N_{1}^{*}\right)=S S_{M 0}^{4}\left(N_{2}\right)$.

THEOREM D. Suppose that $N_{1}$ and $N_{2}$ are M-recursively saturated countable models of $\mathscr{P} \mathcal{A}$ such that $T h_{M}\left(N_{1}\right)=T h_{M}\left(N_{2}\right)$ and $\operatorname{SS}_{M^{0}}{ }^{0}\left(N_{1}\right) \cong S S_{M}^{\Delta_{0}}\left(N_{2}\right)$. Let $A$ be an arbitrary definable subset of $\left|N_{2}\right|$ such that $D f\left(N_{2},|M|\right) \cap A=\emptyset$. Then there is an elementary embedding $f: N_{1} \rightarrow N_{2}$ which is identical on $M$ and with the property $\operatorname{ran}(f) \cap A=\emptyset$.

THEOREM E. Suppose that $N$ is an M-recursively saturated countable model of $\mathscr{P} \mathcal{A}$. Then $N$ is an elementary extension of $M$ if and only if for each element $b>M$, there is an elementary embedding $f: N_{1} \rightarrow N_{2}$ which is identical on $M$ and with the property $\operatorname{ran}(f)<b$.

Theorem A is a generalization of C. Smorynski's result included in [6]. (See Theorem 2.7 in [6], for reference.) Theorem B is very useful and if we combine this with Theorem $\mathrm{C}$, we have the following result which is closely related to the General Splitting Theorem. (See Theorem 0.17.)

Corollary. Let $N_{1}$ and $N_{2}$ be countable cofinal extensions of $M$. Then $T h_{M}^{\Delta}\left(N_{1}\right)=T h_{M}^{\Delta}\left(N_{2}\right)$ implies $T h_{M}\left(N_{1}\right)=T h_{M}\left(N_{2}\right)$.

Theorems A, B and C will appear in $\S 1$. In theorem D if $A=[b, d]=$ $\{c: b \leqq c \leqq d\}$, we can choose $f$ so that $\operatorname{ran}(f)$ is cofinal with $N$. Theorem $\mathrm{E}$ is an analogy of the result of [4]. Theorems $D$ and $E$ will appear in $\S 3$.

\section{§ 0. Preliminaries.}

Throughout this paper, we use the same symbol for a structure and its universe. $M, N$, and $N_{i}(i=1,2, \cdots)$ are used to denote structures and we usually assume that $M$ is a substructure of $N$ or $N_{i}(i=1,2, \cdots)$. Elements of $M$ are denoted by $a, a_{i}(i=1,2, \cdots)$ and elements of $N$ or $N_{i}(i=1,2, \cdots)$ are denoted by $b, d, e, b_{i}, d_{i}, e_{i}(i=1,2, \cdots)$.

First of all, we introduce two notions $M$-recursiveness and $M$-recursive saturation. The former is a generalization of recursivehess and first introduced by J. Barwise. The latter is a generalization of recursive saturation. To explain these notions, we need the notion of hereditarily finite set over $M$. (See [1], 
for reference.)

0.1. Definition. Let $A$ be a set. Then $\mathrm{HF}_{A}$ is the set of hereditarily finite sets over $A$. The explicit definition is as follows:

$\mathrm{HF}_{A}(0)=\emptyset$,

$\mathrm{HF}_{A}(n+1)=$ the set of all finite subset of $\mathrm{HF}_{A}(n) \cup A$,

$\mathrm{HF}_{A}=$ the union of all $\mathrm{HF}_{A}(n)$ 's.

If $A$ is the empty set, we omit $A$ in the above definitions. If $M$ is a structure, $\mathbf{H F}_{M}$ denotes the structure $\left(M ; \mathrm{HF}_{M}, \in\right)$.

0.2. Definition. Let $L \cong \mathbf{H F}$ be a finite language and $M$ an $L$-structure. Then $L(M)$ is the language obtained from $L$ by the addition of new constant $c_{a}=\langle a, \emptyset\rangle$ for each $a \in M$. i. e.,

$$
L(M)=L \cup\{\langle a, \emptyset\rangle: a \in M\} .
$$

0.3. Definition. Let $A$ be a subset of $\mathbf{H F}_{M}$. Then

i) $A$ is $M$-recursive iff $A$ is $\Delta_{1}$ on $\mathbf{H F}_{M}$,

ii) $A$ is $M$-recursively enumerable iff $A$ is $\Sigma_{1}$ on $\mathbf{H F}_{M}$.

We denote the set of all formulas formulated in $L(M)$ by $L(M)^{*} . \quad L(M)^{*}$ is clearly an $M$-recursive subset of $\mathbf{H F}_{\boldsymbol{M}}$.

0.4. Definition. Let $M$ and $N$ be structures for a finite language $L$ such that $M \cong N$. Let $\tau\left(x, y_{1}, \cdots, y_{n}\right)$ be a subset of $L(M)^{*}$ and $b_{1}, \cdots, b_{n}$ elements of $N$. Then we say $\tau\left(x, c_{b_{1}}, \cdots, c_{b_{n}}\right)$ is an $L(M)$-type over $N$ if it is finitely satisfiable in $(N, b)_{b \in N}$, i. e.,

$$
(N, b)_{b \in N} \vDash \exists x W \tau_{0}\left(x, c_{b_{1}}, \cdots, c_{b_{n}}\right),
$$

for every finite subset $\tau_{0}$ of $\tau$. An $L(M)$-type $\tau\left(x, c_{b_{1}}, \cdots, c_{b_{n}}\right)$ over $N$ is said to be an $M$-recursive type (M-recursively enumerable type) if $\tau\left(x, y_{1}, \cdots, y_{n}\right)$ is $M$-recursive ( $M$-recursively enumerable).

0.5. Definition. Let $M$ and $N$ be as above. Then we say $N$ is $M$-recursively saturated if every $M$-recursive type over $N$ is realized in $N$.

The following theorem can be easily obtained by the elementary chain construction. (See, e.g., [6] for reference.)

0.6. THEOREM. Let $M$ and $N$ be structures for a finite language $L$ with 
$M \cong N$. Then there is an $M$-recursively saturated elementary extension $N^{*}$ of $N$ having the same cardinality as $N$.

The following theorem will give us some information concerning the relation between recursive saturation and $M$-recursive saturation.

0.7. THEOREM. If $N$ is $M$-recursively saturated, then $N$ is recursively saturated.

Proof. It is clear that $\omega \subseteq \mathbf{H F}$, and a subset $A$ of $\omega$ is recursive iff it is $\Delta_{1}$ on $\mathbf{H F}$. Since $\mathbf{H F}$ is $\Delta_{1}$ on $\mathbf{H F}_{\boldsymbol{M}}$, every recursive set is $\Delta_{1}$ on $\mathbf{H F}_{\boldsymbol{M}}$. Thus every recursive type $\tau$ over $N$ is realized in $N$, if $N$ is $M$-recursively saturated.

It is well-known that if $N$ is recursively saturated, then $N$ realizes every recursively enumerable type over $N$. The following theorem is a generalization of this fact and the idea of the proof is analogous to that of Theorem 4.13 of [2].

0.8. THEOREM. If $N$ is $M$-recursively saturated, then $N$ realizes every $M$-recursively enumerable type over $N$.

ProOF. Let $c_{x}=\langle x, \emptyset\rangle$ be an $M$-recursive function which gives a new constant of $L(M)^{*}$ corresponding to $x \in M$. Let $\operatorname{sub}(x)$ be an $M$-recursive function defined on $L(M)^{*}$ which gives the set of subformulas of $x$. Using these functions, we define three formulas $\mathrm{Eq}(x), \operatorname{And}(x)$ and $H$-And $(x)$ by:

$\mathrm{Eq}(x)=" x$ is a sentence of the form $c_{y}=c_{y}$ for some $y$ ",

$\operatorname{And}(x)=\operatorname{Eq}(x) \vee " x$ is the sentence $\forall v_{0}\left(v_{0}=v_{0}\right)$ "

$\vee \exists y, z \in \operatorname{sub}(x)(\neg \mathrm{Eq}(y) \wedge$ " $x$ is the sentence $y \wedge z$ "),

$H$-And $(x)=\operatorname{And}(x) \wedge \forall y \in \operatorname{sub}(x) H-\operatorname{And}(y)$.

Clearly, they are $\Delta_{1}$-formulas of set theory. Next we define a function $f$ by:

$$
\begin{aligned}
& f\left(c_{a}=c_{a}\right)=a, \\
& f\left(\forall v_{0}\left(v_{0}=v_{0}\right)\right)=\emptyset, \\
& f(x \wedge y)=f(x) \cup\{f(y)\} .
\end{aligned}
$$

Then $\operatorname{ran}(f)=\mathbf{H F}_{M}$, and $f$ can be expressed by a $\Delta_{1}$-formula. Let $\tau\left(x, c_{b_{1}}, \cdots, c_{b_{n}}\right)$ be an $M$-recursively enumerable type over $N$ and let $\exists z D(y, z)$ be a $\Sigma_{1}$-formula which defines $\tau\left(x, y_{1}, \cdots, y_{n}\right)$ on $\mathbf{H F}_{M}$. Let $D^{*}(x)$ denote the following $\Delta_{1}$-formula :

$\exists y, z \in \operatorname{sub}(x)(D(y, f(z)) \wedge$ “ $x$ is the sentence $y \wedge z " \wedge H$-And $(z))$. 
Then $D^{*}$ defines a certain set $\tau^{*}\left(x, y_{1}, \cdots, y_{n}\right) \subseteq L(M)^{*}$ on $\mathbf{H F}_{M}$. Evidently, $\tau^{*}\left(x, c_{b_{1}}, \cdots, c_{b_{n}}\right)$ is an $M$-recursive type over $N$ and it is realized by some $b \in N$. Now it is clear this $b$ also realizes $\tau\left(x, c_{b_{1}}, \cdots, c_{b_{n}}\right)$ in $N$.

In the remainder of this paper, we shall concentrate on the study of countable models of $\mathcal{P} \mathcal{A}$. We fix some finite language $L \cong \mathbf{H F}$ which contains 0,1 , ,$+ \cdot$. We assume that $\mathscr{P} A$ is formulated in $L$, i. e., $\mathcal{Q} A$ is a 1 -st order Peano arithmetic with the induction schema for every formula of $L . M, N$ and $N_{i}$ $(i=1,2, \cdots)$ are used to denote countable models of $\mathcal{P} \mathcal{A}$. We usually identify $(N, b)_{b \in N}$ with $N$ itself.

0.9. Definition. Let $M \cong N$. Then we say:

i) $N$ is an end extension of $M\left(M \subseteq_{e} N\right)$ iff every element of $N$ which is less than some element of $M$ actually belongs to $M$,

ii) $N$ is a confinal extension of $M\left(M \leqq{ }_{c} N\right)$ iff for each element $b \in N$, there is an element $a \in M$ such that $b<a$.

$M \prec{ }_{e} N$ and $M \prec_{c} N$ mean the elementary end extension and the elementary cofinal extension, respectively.

0.10. Definition. Let $M \subseteq N$. Then :

i) $N$ is $M$-short iff there is an element $b \in N$ such that every element $d \in N$ is less than some $e \in N$ which is definable with parameters from $M \cup\{b\} . N$ is $M$-tall iff $N$ is not $M$-short.

ii) An $L(M)$-type $\tau\left(x, c_{b_{1}}, \cdots, c_{b_{n}}\right)$ over $N$ is short iff $\tau$ contains a formula of the form $x<c_{b}$ for some $b \in M \cup\left\{b_{1}, \cdots, b_{n}\right\}$.

iii) $N$ is $M^{s}$-recursively saturated iff $N$ realizes every short $M$-recursive type.

0.11. Definition. A function $\left\ulcorner_{*}\right\urcorner^{M}: L(M) \rightarrow M$ is called a coding function of $L(M)^{*}$ if it suffices the following conditions:

i) $\left\ulcorner_{*}\right\urcorner^{M}$ is one-one and $M$-recursive,

ii) $\ulcorner\phi\urcorner^{M}>\left\ulcorner\phi_{0}\right\urcorner^{M}$ for every $\phi \in L(M)^{*}$ and every subformula $\phi_{0}$ of $\phi$.

Coding functions do exist. Moreover, if $M \subseteq N,\left\ulcorner_{*}\right\urcorner^{M}$ can be taken as a restriction of $\ulcorner *\urcorner^{N}$ to $L(M)^{*}$. In this context, we usually write $\ulcorner *\urcorner$ instead of $\ulcorner *\urcorner\urcorner^{M}$.

0.12. Definition. Let $M \subseteq N, A \subseteq N$ and $\Gamma \subseteq L(M)^{*}$. Then $S_{M}^{T}(N, A)$ is the set which is defined by: 
$S S_{\mathcal{M}}^{\Gamma}(N, A)=\{X \cap M: X$ is a subset of $N$ which is definable in $N$ using a $\Gamma$-formula with parameters from $A\}$. If $\Gamma=L(M)^{*}$ we omit it, if $A=N$ we omit it, if $A=N$ we omit it. If $Y \leqq L(M)^{*}$, we use the expression $Y \in{ }^{*} S S_{\mathcal{M}}(N)$ to denote the relation: There is a set $Y^{*} \in S S_{M}(N)$ such that $Y=\left\{\phi:\ulcorner\phi\urcorner \in Y^{*}\right\}$.

It is clear that if there is an element $b>M, S S_{M}(N)$ and $S S_{M}^{A} 0(N)$ determine the same set.

0.13. Definitionn. Let $M \cong N$ and $\Gamma \leqq L(M)^{*}$. Then we put:

i) $T h_{M}(N)=\left\{\phi \in L(M)^{*}: N \vDash \phi\right\}$,

ii) $T h_{M}^{\Gamma}(N)=\left\{\phi\left(c_{a_{1}}, \cdots, c_{a_{n}}\right): a_{1}, \cdots, a_{n} \in M, \phi \in \Gamma\right.$ and $\left.N \vDash \phi\left(c_{a_{1}}, \cdots, c_{a_{n}}\right)\right\}$.

0.14. Definition. Let $\Gamma$ be a subset of $L(M)^{*}$. A formula $\operatorname{Tr}_{\Gamma}(x, y)$ is said to be a truth definition for $\Gamma$ in $M$ if for each $\phi\left(x_{1}, \cdots, x_{n}\right) \in \Gamma$ and each $a \in M$,

$$
M \vDash \operatorname{Tr}_{\Gamma}\left(\ulcorner\phi\urcorner, c_{a}\right) \leftrightarrow \phi\left(\left(c_{a}\right)_{1}, \cdots,\left(c_{a}\right)_{n}\right),
$$

where $(x)_{y}$ is the $y$-th index of the binary expansion of $x$.

$\Delta_{0}(M)$ is the set of formulas in $L(M)^{*}$ which have only bounded quantifiers. $\Sigma_{n}(M)$ is the set of formulas in $L(M)^{*}$ which have form $\exists x_{1} \forall x_{2} \exists x_{3} \forall x_{4} \cdots Q_{n} x_{n} \phi$, where $\phi$ is a formula in $\Delta_{0}(M)$. It can be easily shown that there is a truth definition for $\Sigma_{n}(M)$ in $M$ for each $n \in \omega$. The reader who is not familiar with the properties of truth definitions can refer to $\S 3$ of Chapter 1 in [8].

0.15. Definition. Let $M$ be a common submodel of $N_{1}$ and $N_{2}$. Then:

i) A partial function $f: N_{1} \rightarrow N_{2}$ is said to be M-identical iff $f$ is identical on $M$,

ii) A partial function $f: N_{1} \rightarrow N_{2}$ is said to be a partial elementary embedding iff $f$ is a restriction of some elementary embedding $g: N_{1} \rightarrow N_{2}$,

iii) $\operatorname{Emb}\left(N_{1}, N_{2} ; M\right)=$ the set of $M$-identical elementary embeddings of $N_{1}$ into $N_{2} . \quad P-\operatorname{Emb}\left(N_{1}, N_{2} ; M\right)=$ the set of partial $M$-identical elementary embeddings of $N_{1}$ into $N_{2}$ such that $\operatorname{dom}(f)-M$ is finite. $\operatorname{Isom}\left(N_{1}, N_{2} ; M\right)=$ the set of $M$-identical isomorphisms of $N_{1}$ onto $N_{2}$.

Before beginning the study of models of arithmetic, we must state the Elementary Splitting Theorem and the General Splitting Theorem. The latter is, of course, an extension of the former.

0.16. Theorem. (Elementary Splitting Theorem) Let $M \prec N$. Then there is another model $M^{*}$ such that $M \prec_{c} M^{*} \prec_{e} N$. 
0.17. Theorem. (General Splitting Theorem by Gaifman) Let $N$ be a $\Delta_{0}$-elementary extension of $M$. Then there is another model $M^{*}$ such that $M \prec_{c} M^{*} \subseteq_{e} N$.

\section{$\S 1$. Isomorphism conditions.}

In $\S 0$ we showed that if $N$ is $M$-recursively saturated, it realizes every $M$-recursively enumerable type. In case $N$ is a model of arithmetic, the following more useful result holds. The proof is almost the same as that of Theorem 1.12 of [6].

1.1. PROPOSITION. Suppose that $N$ is $M$-recursively saturated ( $M^{s}$-recursively saturated). Then every (short) $L(M)$-type $\tau\left(x, c_{b_{1}}, \cdots, c_{b_{n}}\right) \in * S S_{M}(N)$ is realized in $N$.

This proposition will be used freely without any mention. The following theorem is also a generalization of C. Smorynski's result included in [6].

1.2. THEOREM. Suppose that $N_{1}$ and $N_{2}$ are M-recursively saturated. Then the following three conditions are equivalent:

i) $\operatorname{Isom}\left(N_{1}, N_{2} ; M\right) \neq \emptyset$,

ii) $T h_{M}\left(N_{1}\right)=T h_{M}\left(N_{2}\right)$ and $S S_{M}\left(N_{1}\right)=S S_{M}\left(N_{2}\right)$,

iii) $T h_{M}\left(N_{1}\right)=T h_{M}\left(N_{2}\right)$ and $S_{M}^{\Delta} 0\left(N_{1}\right)=S S_{M}^{\Delta} 0\left(N_{2}\right)$.

The following example shows that Theorem 1.2 fails if we assume only the $M^{s}$-recursive saturation of $N_{1}$ and $N_{2}$.

1.3. REMARK. Let $M$ be an arbitrary model of $\mathcal{P} \mathcal{A}$. Then there are nonisomorphic elementary extensions $N_{1}$ and $N_{2}$ of $M$ such that

i) $N_{1}$ and $N_{2}$ are $M^{s}$-recursively saturated, and

ii) $S S_{M}\left(N_{1}\right)=S S_{M}\left(N_{2}\right)$.

The existence of such $N_{1}$ and $N_{2}$ can be shown by the method similar to the one used in the proof of Theorem 3.9 in [5]. In spite of Remark 1.3, the following form of isomorphism conditions hold.

1.4. THEOREM. Suppose that $N_{1}$ and $N_{2}$ are cofinal extensions of $M$. If $N_{1}$ and $N_{2}$ are $M^{s}$-recursively saturated, the following three conditions are equivalent:

i) $\operatorname{Isom}\left(N_{1}, N_{2} ; M\right) \neq \emptyset$,

ii) $T h_{M}\left(N_{1}\right)=T h_{M}(N)$ and $S S_{M}\left(N_{1}\right)=S S_{M}\left(N_{2}\right)$, 
iii) $T h_{M}^{\Delta}{ }^{0}\left(N_{1}\right)=T h_{M}^{\Delta} 0\left(N_{2}\right)$ and $S S_{M^{0}}^{\Delta}\left(N_{1}\right)=S S_{M}^{\Delta} 0\left(N_{2}\right)$.

RRoof. The implications i) $\Rightarrow$ ii) and i) $\Rightarrow$ iii) are immediate. The proofs of the implications ii $\Rightarrow$ i) and iii) $\Rightarrow$ i) are similar and so we prove only the implication iii) $\Rightarrow$ i). Let $N_{1}-M=\left\{b_{i}\right\}_{i \in \omega}, \quad N_{2}-M=\left\{d_{i}\right\}_{i \in \omega}$. We construct partial isomorphisms $f_{n}$ by induction so that for all $n \in \omega$,

a) $f_{n} \cong f_{n+1}, \operatorname{dom}\left(f_{n}\right) \supseteqq M \cup\left\{b_{i}\right\}_{i<n}$ and $\operatorname{ran}\left(f_{n}\right) \supseteqq M \cup\left\{d_{i}\right\}_{i<n}$,

b) for every $\phi\left(x_{1}, \cdots, x_{m}\right) \in \Delta_{0}(M)$ and every $e_{1}, \cdots, e_{m} \in \operatorname{dom}\left(f_{n}\right)$, if $N_{1} \vDash$ $\phi\left(c_{e_{1}}, \cdots, c_{e_{m}}\right)$ then $N_{2} \vDash \phi\left(c_{f_{n}\left(e_{1}\right)}, \cdots, c_{f_{n}\left(e_{m}\right)}\right)$.

We put $f_{0}=i d_{M}$. Then $f_{0}$ satisfies the condition b) by the assumption $\operatorname{Th}_{M}^{\Delta^{0}}\left(N_{1}\right)$ $=T h_{M}^{\Delta}{ }^{\Delta}\left(N_{2}\right)$. We assume that $f_{n}$ is already defined. We shall specify the image of $b_{n}$ and the inverse image of $d_{n}$. Let $\tau\left(x, y_{1}, \cdots, y_{m}\right)=\left\{\phi \in \Delta_{0}(M): N_{1} \vDash\right.$ $\left.\phi\left(c_{b_{n}}, c_{b_{1}^{\prime}}, \cdots, c_{b_{m}^{\prime}}\right)\right\}$, where $\left\{b_{1}^{\prime}, \cdots, b_{m}^{\prime}\right\}=\operatorname{dom}\left(f_{n}\right)-M$. Choose $a$ from $M$ with $a>b_{n}$ and $\phi_{0}, \cdots, \phi_{p} \in \tau$. Then $\exists x<c_{a} \underset{i \leq p}{W} \phi_{i}\left(x, c_{b_{1}^{\prime}}, \cdots, c_{b_{m}^{\prime}}\right)$ holds in $N_{1}$, so by the induction hypothesis, $\exists x<c_{a} \underset{\imath \leq p}{W} \phi_{i}\left(x, c_{f_{n}\left(b_{1}^{\prime}\right)}, \cdots, c_{f_{n}\left(b_{m}^{\prime}\right)}\right)$ holds in $N_{2}$. This shows that $\tau\left(x, c_{f_{n}\left(b_{1}^{\prime}\right)}, \cdots, c_{f_{n}\left(b_{m}^{\prime}\right)}\right)$ is a short $L(M)$-type over $N_{2}$. Since there is truth definition for $\Delta_{0}(M)$-formulas, $\tau\left(x, c_{f_{n}\left(b_{1}^{\prime}\right)}, \cdots, c_{f_{n}\left(b_{m}^{\prime}\right)}\right) \in * S S_{M^{4}}^{\Delta_{0}}\left(N_{2}\right)$. So that this type is realized by some $d^{*} \in N_{2}$. In the same way we choose $b^{*} \in N_{1}$ corresponding to $d_{n}$. Finally, we put $f_{n+1}=f_{n} \cup\left\{\left\langle b_{n}, d^{*}\right\rangle,\left\langle b^{*}, d_{n}\right\rangle\right\}$. Then $f=$ $\bigcup_{n \in \boldsymbol{\omega}} f_{n}$ is the desired isomorphism.

1.5. COROLlary. Suppose that $N_{1}$ and $N_{2}$ are $M^{s}$-recursively saturated extensions of $M$ with $T h_{M}^{4} 0\left(N_{1}\right)=T h_{M}^{\Delta} 0\left(N_{2}\right)$ and $M \cong{ }_{c} N_{1}$. If $N_{1}$ and $N_{2}$ satisfy the condition:

$$
S S_{M}^{\Delta} 0\left(N_{1}\right)=S S_{M}^{\Delta} 0\left(N_{2}, N_{2}^{*}\right),
$$

then there is an M-identical isomorphism $f$ of $N_{1}$ onto $N_{2}^{*}$, where $N_{2}^{*}=\left\{b \in N_{2}: b\right.$ is less than some $a \in M\}$.

As mentioned earlier, if $M \cong N$, then $N$ can be elementarily extendable to an $M$-recursively saturated model. Now we state some theorems concerning about extendability.

1.6. Theorem. Suppose that $N$ is M-tall (M-short). Then there is an elementary cofinal extension $N^{*}$ of $N$ such that $N^{*}$ is M-recursively satrated (respectively, $M^{s}$-recursively saturated).

Proof. Let $N^{\prime}$ be an $M$-recursively saturated elementary extension of $N$. 
Then, using the Elementary Splitting Theorem, we have $N^{*}$ such that $N \prec{ }_{c} N^{*}$ $\prec_{e} N^{\prime}$. This $N^{*}$ is the desired one.

1.7. THEOREM. Let $N_{1}, N_{2} \supseteq M$. Theu there are M-recursively saturated elementary extensions $N_{1}^{*}$ of $N_{1}$ and $N_{2}^{*}$ of $N_{2}$ such that $\operatorname{SS}_{M}\left(N_{1}^{*}\right)=S S_{M}\left(N_{2}^{*}\right)$.

1.8. THEOREM. Suppose that $N_{1}$ and $N_{2}$ are cofinal extensions of $M$ with $T h_{M}^{4}\left(N_{1}\right)=T h_{M}^{4}\left(N_{2}\right)$. Then there are $M^{s}$-recursively saturated elementary cofinal extensions $N_{1}^{*}$ of $N_{1}$ and $N_{2}^{*}$ of $N_{2}$ such that $\operatorname{SS}_{M}^{0}\left(N_{1}^{*}\right)=S S_{M}^{A_{0}}\left(N_{2}^{*}\right)$.

The proofs of the above two theorems are similar. So we give a proof only for the one that seems more difficult.

Proof OF THEOREM 1.8. Let $S S_{M}^{\Delta}\left(N_{1}\right)=\left\{X_{i}\right\}_{i \in \omega}$ and $C=\left\{c_{i}\right\}_{i \in \omega}$ (a set of new constants). For each $X_{i}$, choose $\phi_{i} \in \Delta_{0}(M), b_{i} \in N_{1}$ and $a_{i} \in M$ such that $X_{i}=\left\{a \in M: N_{1} \vDash T \gamma_{0}\left(\ulcorner\phi\urcorner,\left\langle b_{i}, c_{a}\right\rangle\right)\right\}$ and $\left\langle\left\ulcorner\phi_{i}\right\urcorner, b_{i}\right\rangle\left\langle a_{i}\right.$, where $T r_{0}$ is a truth definition for $\Delta_{0}(M)$-formulas. We put $T=T h_{N_{2}}\left(N_{2}\right) \cup \bigcup_{i \in \omega}\left\{T r_{0}\left(\left(c_{i}\right)_{1},\left\langle\left(c_{i}\right)_{2}, c_{a}\right\rangle\right): a \in X_{i}\right\}$ $\bigcup_{i \in \omega}\left\{\neg \operatorname{Tr}_{0}\left(\left(c_{i}\right)_{1},\left\langle\left(c_{i}\right)_{2}, c_{a}\right\rangle\right): a \notin X_{i}\right\} \cup \bigcup_{i \in \omega}\left\{c_{i}<c_{a_{i}}\right\}$. Clearly $T$ is a consistent theory. Let $N_{2}^{\prime}$ be a model of $T$. Then $N_{2}^{\prime}$ is an elementary extension of $N_{2}$ with $S S_{M^{0}}^{\Delta_{0}}\left(N_{1}\right) \subseteq S S_{M^{4}}^{\Delta_{0}}\left(N_{2}^{\prime}\right)$. By the Elementary Splitting Theorem, there is another model $N_{2}^{\prime \prime}$ such that $N_{2} \prec{ }_{c} N_{2}^{\prime \prime}$ and $S S_{M}^{4}\left(N_{1}\right) \subseteq S S_{M}^{4}\left(N_{2}^{\prime \prime}\right)$. Now we extend $N_{2}^{\prime \prime}$ to an $M^{s}$-recursively saturated model $N_{2}^{0}$ so that $N_{2} \prec{ }_{c} N_{2}^{0}$ and $S_{M^{0}}{ }^{0}\left(N_{1}\right) \subseteq S S_{M}^{\Delta_{0}}\left(N_{2}^{0}\right)$. Next we construct an $M^{s}$-recursively saturated extension $N_{1}^{0}$ of $N_{1}$ so that $N_{1} \prec{ }_{c} N_{1}^{0}$ and $S S_{M}^{\Delta}{ }^{0}\left(N_{2}^{0}\right) \cong S S_{M}^{4}{ }^{0}\left(N_{1}^{0}\right)$. Iterating these constructions, we obtain elementary chains $\left\{N_{1}^{i}\right\}_{i \in \omega}$ and $\left\{N_{2}^{i}\right\}_{i \in \omega}$ such that for each $n \in \omega$,

a) $N_{1}^{n}$ and $N_{2}^{n}$ are $M^{s}$-recursively saturated,

b) $N_{1}^{n} \prec{ }_{c} N_{1}^{n+1}$ and $N_{2}^{n}<{ }_{c} N_{2}^{n+1}$,

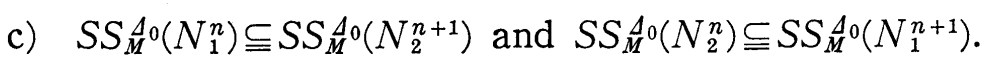

Finally we put $N_{1}^{*}=\bigcup_{i \in \omega} N_{1}^{i}$ and $N_{2}^{*}=\bigcup_{i \in \omega} N_{2}^{i}$. It is a routine to check that $N_{1}^{*}$ and $N_{2}^{*}$ have the desired properties.

Now we apply our results.

1.9. THEOREM. Let $N_{1}$ and $N_{2}$ be cofinal extensions of $M$. Then $T h_{M}^{\Delta}\left(N_{1}\right)$ $=T h_{M}^{\Delta}\left(N_{2}\right)$ implies $T h_{M}\left(N_{1}\right)=T h_{M}\left(N_{2}\right)$.

Proof. Applying Theorem 1.8, we can construct two models $N_{1}^{*}$ and $N_{2}^{*}$ such that 
a) $N_{1}^{*}$ and $N_{2}^{*}$ are $M^{s}$-recursively saturated,

b) $N_{1} \prec{ }_{c} N_{1}^{*}$ and $N_{2} \prec{ }_{c} N_{2}^{*}$,

c) $S S_{M}^{\Delta} 0\left(N_{1}^{*}\right)=S S_{M}^{\Delta} 0\left(N_{2}^{*}\right)$.

Then, by Theorem 1.4, we have

d) $\operatorname{Isom}\left(N_{1}^{*}, N_{2}^{*} ; M\right) \neq \emptyset$.

From this and b), it follows that $T h_{M}\left(N_{1}\right)=T h_{M}\left(N_{2}\right)$.

1.10. Coroltary. Let $N_{1}$ and $N_{2}$ be extensions of $M$ such that $\operatorname{Th}_{M}^{\Delta 0}\left(N_{1}\right)=$ $T h_{M}^{\Delta}\left(N_{2}\right)$. Let $N_{i}^{*}=\left\{b \in N_{i}: b\right.$ is less than some $\left.a \in M\right\} \quad(i=1,2)$. Suppose that $N_{1}^{*}$ and $N_{2}^{*}$ are models of $P A$. Then $T h_{M}\left(N_{1}^{*}\right)=T h_{M}\left(N_{2}^{*}\right)$.

Proof. Since $N_{i}^{*} \subseteq_{e} N_{i} \quad(i=1,2)$, we have $\operatorname{Th}_{M^{\Delta}}^{\Delta}\left(N_{i}\right)=T h_{M}^{\Delta} 0\left(N_{i}^{*}\right) \quad(i=1,2)$. Hence $T h_{M}^{\Delta}{ }^{0}\left(N_{1}^{*}\right)=T h_{M}^{\Delta}{ }^{0}\left(N_{2}^{*}\right)$. By the above theorem, we have the desired property.

The reader should note that we used only the Elementary Splitting Theorem to prove Theorem 1.9 and Corollary 1.10. Corollary 1.10 is closely related to the General Splitting Theorem. But Corollary 1.10 is neither stronger nor weaker than the General Splitting Theorem.

\section{§ 2. Embeddability Conditions.}

In this section, we shall give some theorems concerning embeddability. The main tool of this section is again the back and forth method and so we usually omit the details of the proofs

2.1. Definition. Let $A$ be a structure and $B$ a subset of $A$. Then $\operatorname{Df}(A, B)$ is the set defined by:

$\operatorname{Df}(A, B)=\{a \in A: a$ is definable in $A$ with parameters from $B\}$.

2.2. Proposition. Let $A$ and $B$ as above. Then:

i) $B \subseteq \mathrm{Df}(A, B)$,

ii) $\operatorname{Df}(A, B)=\operatorname{Df}(A, \operatorname{Df}(A, B))$.

First we state a useful lemma, which is interesting of itself.

2.3. LEMMA. Let $N_{1}$ and $N_{2}$ be M-recursively saturated elementary extensions of $M$ with $S S_{M}\left(N_{1}\right) \cong S S_{M}\left(N_{2}\right)$. Suppose that $A$ is a definable subset of $N_{2}$ such that $A \subseteq N_{2}-M$. Then for each $f \in P-\operatorname{Emb}\left(N_{1}, N_{2} ; M\right)$ with $\operatorname{Df}\left(N_{2}, \operatorname{ran}(f)\right)$ 
$\cap A=\emptyset$, and each $b \in N_{1}$, there is an extension $f^{*} \in P-\operatorname{Emb}\left(N_{1}, N_{2} ; M\right)$ of $f$ such that $\operatorname{dom}\left(f^{*}\right)=\operatorname{dom}(f) \cup\{b\}$ and $\operatorname{Df}\left(N_{2}, \operatorname{ran}\left(f^{*}\right)\right) \cap A=\emptyset$.

Proof. Suppose that $\operatorname{dom}(f)=M \cup\left\{b_{1}, \cdots, b_{n}\right\}$ and $\tau\left(x, x_{1}, \cdots, x_{n}\right)=\{\phi(x$, $\left.\left.x_{1}, \cdots, x_{n}\right) \in L(M)^{*}: N_{1} \vDash \phi\left(c_{b}, c_{b_{1}}, \cdots, c_{b_{n}}\right)\right\}$. Then for each finite subset $\tau_{0}$ of $\tau$, the sentence $\exists x M \tau_{0}\left(x, c_{f\left(b_{1}\right)}, \cdots, c_{f\left(b_{n}\right)}\right)$ holds in $N_{2}$. Moreover, if $F_{1}, \cdots, F_{m}$ are $L(M)$-Skolem functions, the following sentence also holds in $N_{2}$ :

$$
\exists x\left(M_{i} \neg \alpha\left(F_{i}\left(x, c_{f\left(b_{1}\right)}, \cdots, c_{f\left(b_{n}\right)}\right)\right) \wedge M \tau_{0}\left(x, c_{f\left(b_{1}\right)}, \cdots, c_{f\left(b_{n}\right)}\right)\right),
$$

where $\alpha$ is the defining formula of $A$ in $N_{2}$. Hence the set $\tau\left(x, c_{f\left(b_{1}\right)}, \cdots, c_{f\left(b_{n}\right)}\right)$ $\cup\left\{\neg \alpha\left(F\left(x, c_{f\left(b_{1}\right)}, \cdots, c_{f\left(b_{n}\right)}\right): F\right.\right.$ an $L(M)$-Skolem function $\}$ is an $L(M)$-type over $N_{2}$ and is realized by some $d \in N_{2}$. If we put $f^{*}=f \cup\{\langle b, d\rangle\}$, then $f^{*}$ is the desired partial elementary embedding.

2.4. THEOREM. Let $N_{1}, N_{2}, M$ and $A$ be as in the above lemma. Then there is an elementary embedding $f \in \operatorname{Emb}\left(N_{1}, N_{2} ; M\right)$ such that $\operatorname{Df}\left(N_{2}, \operatorname{ran}(f)\right) \cap A=\emptyset$.

ProOF. Let $N_{2}-M=\left\{b_{i}\right\}_{i \in \omega}$. We construct partial elementary embeddings $f_{n} \in P-\operatorname{Emb}\left(N_{1}, N_{2} ; M\right)$ by induction so that for all $n \in \omega$,

a) $\operatorname{dom}\left(f_{n}\right)=M \cup\left\{b_{i}\right\}_{i<n}$,

b) $\operatorname{Df}\left(N_{2}, \operatorname{ran}\left(f_{n}\right)\right) \cap A=\emptyset$.

We put $f_{0}=i d_{M}$ and assume that $f_{n}$ is already defined. Using the above lemma, we take $f_{n+1} \in P-\operatorname{Emb}\left(N_{1}, N_{2} ; M\right)$ so that $\operatorname{dom}\left(f_{n+1}\right)=\operatorname{dom}\left(f_{n}\right) \cup\left\{b_{n}\right\}$. Then $f=$ $\bigcup_{n \in \omega} f_{n}$ is the desired elementary embedding.

2.5. COROLLARY. Let $N$ be a $\Delta_{0}$-elementary extension of $M$ and suppose that $N$ is $M$-recursively saturated. Then the following i) and ii) are equivalent:

i) $N$ is an elementary extension of $M$,

ii) For each $b>M$, there is an elementary embedding $f \in \operatorname{Emb}(N, N ; M)$ such that $\operatorname{ran}(f)<b$.

Proof. The implication i) $\Rightarrow$ ii) is immediate by the above theorem. We shall prove only the implication ii) $\Rightarrow \mathrm{i})$. Suppose that $N$ is not an elementary extension of $M$. We only have to show that there is an element $b \in \operatorname{Df}(N, M)$ with $b>M$. By way of contradiction, we assume that there is no such element. Then, by the General Splitting Theorem, $\operatorname{Df}(N, M)$ must be an elementary cofinal extension of $M$. Since $\operatorname{Df}(N, M) \prec N$ is clear, we have $M \prec N$. This is a contradiction. 
In Corollary 2.5 we assumed that $N$ is a $\Delta_{0}$-elementary extension of $M$. The author doesn't know whether this assumption can be eliminated or not.

2.6. THEOREM. Let $N$ be $M$-recursively saturated and suppose that $M<b<d$. If $\operatorname{Df}(N, M) \cap[b, d]=\emptyset$, then there is an elementary embedding $f \in \operatorname{Emb}(N, N ; M)$ such that:

i) $\operatorname{ran}(f) \cap[b, d]=\emptyset$,

ii) $\operatorname{ran}(f)$ is cofinal with $N$.

To prove Theorem 2.6, we need the following lemma.

2.7. Lemma. Let $N$ be an M-recursively saturated extension of $M$. Suppose that $M<b<d$ and $\operatorname{Df}\left(N, M \cup\left\{e_{1}, \cdots, e_{n}\right\}\right) \cap[b, d]=\emptyset$. Then there is an arbitrarily large element $e$ such that $\operatorname{Df}\left(N, M \cup\left\{e, e_{1}, \cdots, e_{n}\right\}\right) \cap[b, d]=\emptyset$.

Proof. Let $e^{*} \in N$ be an arbitrary element. Define the set $\tau(x)$ by :

$$
\tau(x)=\left\{\neg\left(b<F\left(x, e_{1}, \cdots, e_{n}\right)<d\right): F \text { an } L(M) \text {-Skolem function }\right\} \cup\left\{x>e^{*}\right\} .
$$

It is sufficient to prove that $\tau(x)$ is an $M$-recursive type over $N$. The $M$-recursiveness of $\tau(x)$ is clear and so we prove that $\tau(x)$ is finitely satisfiable in $N$. By way contradiction, assume that there are $L(M)$-Skolem functions $F_{1}, \cdots, F_{n}$ such that

$$
N \vDash \forall x>e^{*}\left(\underset{k}{W}\left(b<F_{k}\left(x, e_{1}, \cdots, e_{n}\right)<d\right)\right) .
$$

Now define $\tau^{*}(u, v)$ by :

$$
\begin{aligned}
\tau^{*}(u, v)= & \{a<u<v<b: a \in M\} \\
& \cup\left\{\neg\left(u<F\left(e_{1}, \cdots, e_{n}\right)<v\right): F \text { an } L(M) \text {-Skolem function }\right\} \\
& \cup\left\{\exists y \forall x>y\left(\mathbb{M}_{k}\left(u<F_{k}\left(x, e_{1}, \cdots, e_{n}\right)<v\right)\right\}\right.
\end{aligned}
$$

It is a routine to check that this $\tau^{*}(u, v)$ is an $M$-recursive type over $N$. Suppose that a pair $\left\langle b_{1}, d_{1}\right\rangle$ realizes $\tau^{*}(u, v)$. Then the following hold:

a) $M<b_{1}<d_{1}<b$ and $\operatorname{Df}\left(N, M \cup\left\{e_{1}, \cdots, e_{n}\right\}\right) \cap\left[b_{1}, d_{1}\right]=\emptyset$,

b) $N \vDash \exists y \forall x>y\left(\underset{k}{\mathbb{A}}\left(b_{1}<F_{k}\left(x, e_{1}, \cdots, e_{n}\right)<d_{1}\right)\right)$.

Continueing all these, finally we have a sequence $\left\{\left\langle b_{i}, d_{i}\right\rangle\right\}_{i \in \omega}$ such that for each $i \in \omega$,

c) $M<b_{i+1}<d_{i+1}<b_{i}$,

d) $N \vDash \exists y \forall x>y\left(\underset{k}{\left(M_{k}\right.}\left(b_{i}<F_{k}\left(x, e_{1}, \cdots, e_{n}\right)<d_{i}\right)\right)$. 
But this is impossible and so we conclude that $\tau(x)$ is finitely satisfiable in $N$.

Proof of THEOREM. Let $N-M=\left\{b_{i}\right\}_{i \in \omega}$. We construct partial elementary embeddings $f_{n} \in P-\operatorname{Emb}(N, N ; M)$ so that for each $n \in \omega$,

a) $f_{n} \cong f_{n+1}$ and $\operatorname{dom}\left(f_{2 n}\right) \supseteqq M \cup\left\{b_{i}\right\}_{i<n}$,

b) $\operatorname{Df}\left(N, \operatorname{ran}\left(f_{n}\right)\right) \cap[b, d]=\emptyset$

c) There is an element $e \in \operatorname{ran}\left(f_{2 n+1}\right)$ such that $e>b_{n}$.

The construction of $f_{2 n}$ is the same one that is shown in Theorem 2.4. We shall show only the construction of $f_{2 n+1}$. Suppose that $f_{2 n}$ is already constructed. By the above lemma, there is an element $e>b_{n}$ such that $\operatorname{Df}\left(N, \operatorname{ran}\left(f_{2 n}\right) \cup\{e\}\right)$ $\cap[b, d]=\emptyset$. Then choose an element $e^{*}$ so that $f_{2 n} \cup\left\{\left\langle e^{*}, e\right\rangle\right\}$ will become a partial elementary embedding. Let $f_{2 n+1}$ be this partial elementary embedding. It is clear that $f=\bigcup_{n \in \omega} f_{n}$ is the required one.

QUESTIONS. We state some open questions:

i) In [7], C. Smorynski and J. Stavi proved that recursive saturation is preserved under elementary cofinal extensions. Is $M$-recursive saturation also preserved under elementary cofinal extensions?

ii) Let $N$ be an extension of $M . \quad N$ is said to be $M$-short legged if there is an element $b>M$ such that $\operatorname{Df}(N, M \cup\{b\})-M$ is downward cofinal with $N-M$. Is there any model $N$ which is $M$-recursively saturated and $M$-short legged ?

iii) Let $N_{1}$ and $N_{2}$ be not $M$-short legged and suppose that $T h_{M}\left(N_{1}\right)=$ $T h_{M}\left(N_{2}\right)$. Is it always possible to find another model $N$ and elementary embeddings $f: N_{i} \rightarrow M(i=1,2)$ so that $\operatorname{ran}\left(f_{1}\right)-M$ and $\operatorname{ran}\left(f_{2}\right)-M$ are downward cofinal with each other?

\section{References}

[1] Barwise, J., Admissible sets and structures, Springer-Verlag, Berlin (1975).

[2] Feferman, S., Arithmetization of metamathematics in a general setting, Fundamenta Mathematicae, 49 (1960), 35-92.

[3] Friedman, H., Countable models of set theories, Cambridge Summer School in Mathematical Logic, Lecture Notes in Mathematics, Springer-Verlag, Berlin (1972), 539-537.

[4] Lipshitz, D., Diophantine correct models of arithmetic, Proceedings of the American Mathematical Society, 73 (1979), 107-108.

[5] Smorynski, C., Cofinal extensions of nonstandard models of Arithmetic, Notre Dame Journal of Formal Logic, 22 (1981), 133-144.

[6] Smorynski, C., Recursively saturated nonstandard models of arithmetic, The Journal of Symbolic Logic, 46 (1981), 259-286. 
[7] Smorynski, C. and J. Stavi, Cofinal extension preserves recursive saturation, Model Theory of Algebra and Arithmetic, Lecture Notes in Mathematics, SpringerVerlag, Berlin (1981), 338-345.

[8] Troelstra, A.S., ed., Mathematical Investigation of Intuitionistic Arithmetic and Analysis, Lecture Notes in Mathematics, Springer-Verlag, Berlin (1972).

Department of Mathematics

University of Tsukuba

Sakura-mura, Niihari-gun

Ibaraki, 305 Japan 